Acta Cryst. (1987). C43, 194-197

https://doi.org/10.1107/S010827018709646X

\title{
Hydrogénophosphate de Zinc Hydrate
}

\author{
Par A. Riou, Y. Cudennec et Y. Gérault \\ Laboratoire de Chimie des Matériaux Inorganiques et de Cristallographie, 20 avenue des Buttes de \\ Coësmes, 35043 Rennes CEDEX, France
}

(Reçu le 22 juillet 1986, accepté le 12 septembre 1986)

\begin{abstract}
Zn}_{3}\left[\mathrm{PO}_{3}(\mathrm{OH})\right]_{3} .3 \mathrm{H}_{2} \mathrm{O}, \mathrm{M}_{\mathrm{r}}=538.1$, triclinic, $\mathrm{P}-1, a=6.429(13), b=7.726(11), c=$ 12.285 (16) $\AA, \alpha=74.11$ (10), $\beta=81.35$ (9), $\gamma=80.99$ (9) ${ }^{\circ}, \mathrm{V}=576.0 \AA^{3}, \mathrm{D}_{\mathrm{m}}=3.10$ (1) (by

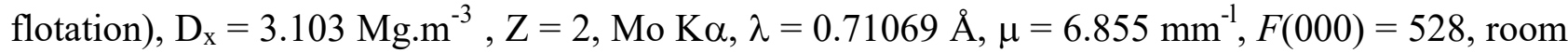
temperature, $\mathrm{R}=0.033$ for 2310 reflections. The phase $\mathrm{Zn}_{3}\left[\mathrm{PO}_{3}(\mathrm{OH})\right]_{3} .3 \mathrm{H}_{2} \mathrm{O}$, has been studied by single-crystal $\mathrm{X}$-ray structure analysis with an automatic diffractometer. The structure contains $\left[\mathrm{ZnO}_{6}\right]$ and $\left[\mathrm{ZnO}_{5}\right]$ polyhedra linked together by $\left[\mathrm{PO}_{3}(\mathrm{OH})\right]$ tetrahedra to build complex sheets in the $a b$ plane. All these sheets are linked only by hydrogen bonds.
\end{abstract}

\section{Introduction}

De nombreux travaux ont été consacrés à l'étude chimique et structurale des phosphates de zinc. Dans une étude récente du système ternaire $\mathrm{ZnO}, \mathrm{P}_{2} \mathrm{O}_{5}, \mathrm{H}_{2} \mathrm{O}$ (Cudennec, Lecerf, Riou \& Gérault, 1985), comportant un rappel bibliographique des travaux antérieurs, nous avons obtenu par synthèse hydrothermale quelques uns des phosphates déjà cités dans la littérature chimique et présenté un nouvel hydrate de formule $\mathrm{Zn}_{3}\left(\mathrm{PO}_{4}\right)_{2} \cdot \mathrm{H}_{2} \mathrm{O}$. Parmi ces composés on trouve en particulier des hydrogénophosphates de formule $\mathrm{Zn}\left[\mathrm{PO}_{3}(\mathrm{OH})\right]$. $\mathrm{xH}_{2} \mathrm{O}$ qui à notre connaissance n'ont jamais fait l'objet d'études structurales. Seul un mémoire comporte une détermination des paramètres cristallins de $\mathrm{Zn}\left[\mathrm{PO}_{3}(\mathrm{OH})\right] . \mathrm{H}_{2} \mathrm{O}$ (Kovarskii, Kozina, Lepilina \& Fomina, 1971) mais les résultats sont erronés.

Un isomère de $\mathrm{Zn}\left[\mathrm{PO}_{3}(\mathrm{OH})\right] . \mathrm{H}_{2} \mathrm{O}$ présenté sous la formule $\mathrm{H}_{3} \mathrm{OZnPO}_{4}$ a fait l'objet d'une étude structurale (Sandomirskii, Klientova, Simonov \& Belov, 1977), mais les auteurs fournissent peu d'information sur la préparation du cristal étudié. Dans l'étude chimique précédemment citée, nous indiquons les raisons qui permettent d'avancer l'hypothèse que les cristaux étudiés correspondent très probablement à $\mathrm{NH}_{4} \mathrm{ZnPO}_{4}$ et non à $\mathrm{H}_{3} \mathrm{OZnPO}_{4}$. Compte tenu de la diversité des résultats présentés dans les travaux antérieurs et de leurs contradictions, il nous a paru intéressant d'effectuer 
la détermination structurale complète du composé $\mathrm{Zn}\left[\mathrm{PO}_{3}(\mathrm{OH})\right] \cdot \mathrm{H}_{2} \mathrm{O}$ qui est en fait un trimère de formule $\mathrm{Zn}_{3}\left[\mathrm{PO}_{3}(\mathrm{OH})\right]_{3}$. $3 \mathrm{H}_{2} \mathrm{O}$. Ce travail est exposé dans ce mémoire.

\section{Partie expérimentale}

Cristaux de $\mathrm{Zn}_{3}\left[\mathrm{PO}_{3}(\mathrm{OH})\right]_{3} .3 \mathrm{H}_{2} \mathrm{O}$ obtenus par une très lente évaporation à $353 \mathrm{~K}$ d'un système comprenant $30 \%$ de $\mathrm{P} 2 \mathrm{O} 5,17 \%$ de $\mathrm{ZnO}$ et $53 \%$ d'eau. Ces cristaux précipitent sous forme d'aiguilles transparentes parallélépipédiques tronquées.

Etudes préliminaires radiocristallographiques menées sur chambres photographiques de Weissenberg et de précession. Paramètres de la maille cristalline affinés par moindres carrés à partir de 25 réflexions optimisées sur diffractomètre automatique. Enregistrement des intensités diffractées sur un diffractomètre automatique (Enraf-Nonius); monochromateur: lame de graphite; distance cristal-détecteur: $173 \mathrm{~mm} ; 1<0<27^{\circ}$; balayage: $\omega$ - $2 \theta$; amplitude de balayage $(1,00+035$ $\operatorname{tg} \theta)^{\circ}$; ouverture $(2,00+0,45 \operatorname{tg} \theta)^{\circ} .2518$ réflexions indépendantes mesurées, corrigées du facteur de Lorentz-polarisation mais pas de correction d'absorption; dimensions du cristal: 825 x $125 \times 50$ $\mu \mathrm{m}$; pas de variations significatives des trois réflexions de référence; h:-8 à 8 ; k:-9 à 9 ; $1: 0$ à 15 . 2310 réflexions avec I $>\sigma(\mathrm{I})$ conservées pour la résolution de la structure. Structure résolue par la méthode de l'atome lourd: positions des atomes de zinc et de phosphore déterminées par déconvolution de la fonction de Patterson tridimensionnelle. Positionnement des atomes d'oxygène appartenant aux groupements phosphates et aux molécules d'eau à l'aide des cartes de densités électroniques tridimensionnelles. Affinement de la structure par une méthode de moindres carrés à matrice totale et minimisant la fonction $\Sigma w(\Delta F)^{2}$; valeur de 1a pondération: 0,040. Première série d'affinement des positions et des coefficients d'agitation thermique isotropes puis anisotropes. Positions des atomes d'hydrogène obtenues alors par synthèse 'différence'. Seconde série d'affinement incluant les 30 atomes indépendants de la structure; 218 variables affinées; indice résiduel $\mathrm{R}=3,3 \%$ et $w \mathrm{R}=4,4 \%$; coefficient d'extinction secondaire: $6,6738 \times 10^{-7}$; erreur dans l'observation de poids unitaire: 1,$96 ;|\Delta \rho|<1$ e $\AA^{-3}$. Facteurs de diffusion utilisés: Cromer \& Waber (1965). Calculs effectués sur PDP 11/60 avec la bibliothèque de programmes cristallographiques SDP (Frenz,1978).

\section{Discussion}

Le Tableau 1 rassemble les valeurs finales des positions atomiques avec leurs écarts types ainsi que les facteurs d'agitation thermique isotrope équivalents*. Conformément à la formule proposée, la structure de $\mathrm{Zn}_{3}\left[\mathrm{PO}_{3}(\mathrm{OH})\right]_{3}$. $3 \mathrm{H}_{2} \mathrm{O}$ (Fig.1) contient trois atomes de zinc et de phosphore indépendants. Les atomes de phosphore possèdent un environnement tétraédrique d'oxygènes. Les atomes de zinc présentent des environnements différents: $\mathrm{Zn}(1)$ et $\mathrm{Zn}(2)$ sont à coordinence octaédrique alors que $\mathrm{Zn}(3)$ possède cinq oxygènes dans son proche environnement.

Le Tableau 2 donne les valeurs des principaux angles et distances de liaisons dans les divers polyèdres. Chacun des trois atomes de phosphore indépendant $\mathrm{P}(1), \mathrm{P}(2)$ et $\mathrm{P}(3)$ est entouré par trois atomes d'oxygène et un hydroxyle $\mathrm{OH}$, formant ainsi un tétraèdre de formule $\left[\mathrm{PO}_{3}(\mathrm{OH})\right]^{2-}$. Les longueurs de liaison P-O sont assez comparables lorsque l'oxygène n'est pas directement lié à un atome d'hydrogène; la longueur moyenne de ces liaisons est alors de 1,525 $\AA$, ce qui correspond bien aux valeurs habituellement observées. On constate en revanche une sensible augmentation de la longueur de liaison P-O lorsque l'oxygène: $0(6), \mathrm{O}(7)$ ou $\mathrm{O}(12)$ appartient à un groupement hydroxyle $\mathrm{OH}$, la valeur moyenne observée dans ce cas est de 1,579 Å. Ce phénomène a déjà été observé dans le cas d'autres hydrogénophosphates, notamment dans la structure de $\mathrm{Cu}\left[\mathrm{PO}_{3}(\mathrm{OH})\right] \cdot \mathrm{H}_{2} \mathrm{O}$ (Boudjada, 1980). Les angles de liaison O-P-O subissent des écarts non négligeables par rapport à l'angle théorique, l'écart maximum étant de $6,5^{\circ}$. Les atomes d'oxygène non liés à l'hydrogène appartenant aux trois groupements phosphate, n'ont pas tous le même 
environnement: ceux appartenant aux deux tétraèdres $\left[\mathrm{P}(\mathrm{l}) \mathrm{O}_{3}(\mathrm{OH})\right]$ et $\left[\mathrm{P}(2) \mathrm{O}_{3}(\mathrm{OH})\right]$ sont tous partagés avec deux atomes de zinc alors que $\mathrm{O}(9)$ et $\mathrm{O}(10)$ appartenant à $\left[\mathrm{P}(3) \mathrm{O}_{3}(\mathrm{OH})\right]$ sont partagés avec un seul atome de zinc, et que $\mathrm{O}(11)$ appartenant aussi à $\left[\mathrm{P}(3) \mathrm{O}_{3}(\mathrm{OH})\right]$ n'est lié à aucun zinc. Cette propriété distingue $\left[\mathrm{P}(3) \mathrm{O}_{3}(\mathrm{OH})\right]$ des autres tétraèdres.

L'atome de zinc $\mathrm{Zn}(1)$ possède un environnement octaédrique irrégulier de six oxygènes, les longueurs de liaisons Zn-O sont en effet comprises entre 2,002 $\AA$ et 2,265 $\AA$. Les angles O-Zn(1)-O subissent par ailleurs d'importants écarts par rapport à ceux de l'octaèdre régulier. A noter que $\mathrm{Zn}(1)$ n'est lié directement à aucune molécule d'eau. $\mathrm{Zn}(2)$ qui occupe aussi le centre d'un octaèdre irrégulier de formule $\left[\mathrm{Zn}(2) \mathrm{O}_{5} \mathrm{O} w\right]$ est lié directement à une molécule d'eau. Les longueurs de liaisons $\mathrm{Zn}-\mathrm{O}$ subissent d'importantes variations qui sont comprises entre 2,009 $\AA$ et 2,383 $\AA$ comme dans le cas de l'octaèdre précédent les écarts angulaires sont importants. $\mathrm{Zn}(3)$ possède un environnement pentaédrique de formule $\left[\mathrm{Zn}(3) \mathrm{O}_{3}(\mathrm{O} w)_{2}\right]$. Ce pentaèdre est formé par un plan carré d'oxygènes: $\mathrm{O}(3), \mathrm{O}(8), \mathrm{O} w(1), \mathrm{O} w(2)$ liés au zinc par des liaisons de longueurs variant entre 1,981 et 2,202 Å auxquels s'ajoute un cinquième oxygène: $\mathrm{O}(9)$ situé dans une direction perpendiculaire au plan.des oxygènes précédents. L'atome $\mathrm{Zn}(3)$ qui se trouve à $1,997 \AA$ de $\mathrm{O}(9)$ est distant de $0,31 \AA$ du plan carré. Le polyèdre de coordination de $\mathrm{Zn}(3)$ est donc assimilable à une pyramide à base carrée. Un sixième coordinat: $\mathrm{O} w(3)$ diamétralement opposé à $\mathrm{O}(9)$ compléterait un octaèdre très déformé mais sa distance à $\mathrm{Zn}(3): 2,53 \AA$ ne permet pas de le considérer comme véritablement lié à ce dernier.

Les différents polyèdres précédemment décrits mettent en commun des oxygènes, contribuant ainsi à l'enchaînement structural. L'octaèdre $\left[\mathrm{Zn}(1) \mathrm{O}_{6}\right]$ possède deux oxygènes: $\mathrm{O}(\mathrm{l})$ et $\mathrm{O}\left(\mathrm{l}^{\mathrm{i}}\right)$, communs avec un octaèdre voisin du même type, de même il partage deux oxygènes: $O(2)$ et $O(4)$ avec un octaèdre $\left[\mathrm{Zn}(2) \mathrm{O}_{5} \mathrm{O} w\right]$. Ce dernier possède également deux oxygènes: $\mathrm{O}(5)$ et $\mathrm{O}\left(5^{\mathrm{ii}}\right)$, communs avec un octaèdre $\left[\mathrm{Zn}(2) \mathrm{O}_{5} \mathrm{O} w\right]$ de même type. Il résulte de cet assemblage une chaîne octaédrique parallèle à [ $\left[\begin{array}{lll}-1 & 1 & 0\end{array}\right]$ au sein de laquelle alternent par couple, les deux types d'octaèdre.

La liaison inter-chaîne est assurée par les deux groupements $\left[\mathrm{P}(1) \mathrm{O}_{3}(\mathrm{OH})\right]$ et $\left[\mathrm{P}(2) \mathrm{O}_{3}(\mathrm{OH})\right]$, chaque phosphore $\mathrm{P}(1)$ et $\mathrm{P}(2)$ partage en effet, trois de ses oxygènes (non reliés à l'hydrogène) avec les atomes $\mathrm{Zn}(1)$ et $\mathrm{Zn}(2)$ appartenant à deux chaînes voisines. Cet ensemble constitue une trame bidimensionnelle compacte qui se développe à la cote moyenne $\mathrm{z}=1 / 2$. De part et d'autre de cette trame, se trouvent le tétraèdre $\left[\mathrm{P}(3) \mathrm{O}_{3}(\mathrm{OH})\right]$ et le pentaèdre $\left[\mathrm{Zn}(3) \mathrm{O}_{3}(\mathrm{O} w)_{2}\right]$.

L'atome de phosphore $\mathrm{P}(3)$ partage un oxygène: $\mathrm{O}(10)$, avec I'atome de zinc $\mathrm{Zn}(1)$ alors que $\mathrm{Zn}(3)$ met en commun deux de ses oxygènes: $\mathrm{O}(3)$ et $\mathrm{O}(8)$ avec les atomes $\mathrm{Zn}(\mathrm{l})$ et $\mathrm{Zn}(2)$ appartenant à la trame. Les deux polyèdres $\left[\mathrm{P}(3) \mathrm{O}_{3}(\mathrm{OH})\right]$ et $\left[\mathrm{Zn}(3) \mathrm{O}_{3}(\mathrm{O} w)_{2}\right]$ possèdent d'autre part un oxygène commun: $\mathrm{O}(9)$.

L'assemblage de ces polyèdres constitue un feuillet parallèle au plan $a b$. La jonction entre ces feuillets qui se développe à la cote moyenne $z=0$ parallèlement au plan (001) est assurée par des liaisons par pont hydrogène. La présence de ces dernières au sein de la structure est évidente, elles ont été localisées à partir des positions des atomes d'hydrogène, déterminées grâce aux dernières cartes de densité électronique. L'existence de ces liaisons est par ailleurs confirmée par la faible distance entre atomes d'oxygène donneurs et accepteurs mais aussi par le fort décalage des bandes d'absorption des groupements $\mathrm{OH}$ enregistrées sur le spectre infra-rouge.

Dans le Tableau 3 ont été rassemblées les principales informations concernant ces liaisons hydrogène. Ce tableau comporte en outre des indications sur la contribution de ces liaisons hydrogène dans la cohésion de l'édifice structural. On s'aperçoit que seulement le tiers des liaisons hydrogène participe à jonction inter-feuillets, les deux tiers restants sont des liaisons intra-feuillets et servent à la consolidation propre du feuillet (Fig. 2). Parmi les trois atomes d'hydrogène participant à des liaisons inter-feuillets: $\mathrm{H}^{\prime}(\mathrm{O} w 2)$ et $\mathrm{H}^{\prime}(\mathrm{O} w 1)$ proviennent de molécules d'eau; l'une de ces liaisons est assez forte: $\mathrm{O} w(2)-\mathrm{O}(9)=2,70 \AA \AA$; $\mathrm{H}(\mathrm{O} 12)$ qui appartient à l'anion $\left[\mathrm{P}(3) \mathrm{O}_{3}(\mathrm{OH})\right]$ engendre une liaison par pont particulièrement forte puisque $\mathrm{O}(11)-\mathrm{O}(12)=2,63 \AA$. On peut donc considérer que ces liaisons par pont assurent une bonne jonction entre les feuillets. Les autres 
liaisons par pont hydrogène jouent un rôle important dans la consolidation du feuillet notamment dans les zones moins compactes situées en lisière du feuillet; c'est le cas en particulier des liaisons générées à partir de $\mathrm{H}(\mathrm{O} 7), \mathrm{H} "(\mathrm{O} w 1)$ et $\mathrm{H}^{\prime \prime}(\mathrm{O} w 2)$.

Parmi les liaisons par pont hydrogène intra-feuillets, il en existe deux: $\mathrm{O}(7)-\mathrm{H}(\mathrm{O} 7) \ldots \mathrm{O}(11)$ et $\mathrm{O}(6)$ $\mathrm{H}(\mathrm{O} 6) \ldots \mathrm{O}(10)$ exceptionnellement fortes puisque $\mathrm{O}(7)-\mathrm{O}(11)=2,54 \AA$ et $\mathrm{O}(6)-\mathrm{O}(10)=2,58 \AA$. Ces liaisons jouent un rôle particulier puisqu'elles réalisent un pontage entre les trois groupements anioniques indépendants $\mathrm{HPO}_{4}{ }^{2-}$, il existe donc au sein de cette structure un regroupement privilégié des trois hydrogénophosphates, qui compte tenu de la force des liaisons hydrogène, se rapproche d'un trimère anionique de formule $\left[\mathrm{H}_{3} \mathrm{P}_{3} \mathrm{O}_{12}\right]^{6-}$.

* Les listes des facteurs de structure et des paramètres thermiques anisotropes ont été déposées au dépôt d'archives de la British Library document Supply Centre (Supplementary Publication No SUP 43398: 14 pp.). On peut en obtenir des copies en s'adressant à: The Executive Secretary, International Union of Crystallography, 5 Abbey Square, Chester CHI 2HU, Angleterre.

\section{Références}

BoudjadA, A. (1980). Mater. Res. Bull. 15, 1083-1090.

Cromer, D. T. \& W ABer, J. T. (1965). Acta Cryst. 18, 104-109.

Cudennec, Y., Lecerf, A., Riou, A. \& Gerault, Y. (1985). C. $R$. Acad. Sci. Ser. II, 301, 93-98.

Frenz, B. A. (1978). The Enraf-Nonius CAD-4 SDP - A Real-Time System for Concurrent X-ray Data Collection and Crystal Structure Solution. Dans Computing in Crystallography, édité par H. Schenk, R. Olthof-Hazekamp, H. van Koningsveld \& G. C. BAssi, pp. 64-71. Delft Univ. Press.

Kovarski, V. A., Kozina, T. M., Lepilina, R. G. \& Fomina, E. A. (1971). Russ. J. Phys. Chem. 45(8), 1194-1195.

Sandomirskit, P. A., Klientova, G. P., Simonov, M. A. \& Belov, N. V. (1977). Dokl. Akad. Nauk Cryst. 236(3), 597-600. 
Tableau 1. Paramètres atomiques et coefficients d'agitation thermique isotrope équivalents de $\mathrm{Zn}_{3}\left[\mathrm{PO}_{3}(\mathrm{OH})\right]_{3} \cdot 3 \mathrm{H}_{2} \mathrm{O}$

$$
B_{\text {eq }}=\frac{4}{3} \sum_{i} \sum_{j} \beta_{i j} \mathbf{a}_{i} \cdot \mathbf{a}_{j} \text {. }
$$

$\begin{array}{lcccc} & x & y & z & B_{\mathrm{eq}}\left(\AA^{2}\right) \\ \mathrm{Zn}(1) & 0,64777(5) & 0,11454(4) & 0,40954(3) & 0,794(6) \\ \mathrm{Zn}(2) & 0,86031(5) & 0,32728(4) & 0,55015(3) & 0,894(6) \\ \mathrm{Zn}(3) & 0,02054(6) & 0,06425(5) & 0,79939(3) & 1,128(7) \\ \mathrm{P}(1) & 0,8546(1) & 0,8695(1) & 0,63484(6) & 0,61(1) \\ \mathrm{P}(2) & 0,3473(1) & 0,2920(1) & 0,61035(6) & 0,66(1) \\ \mathrm{P}(3) & 0,3245(1) & 0,6930(1) & 0,86479(6) & 0,90(1) \\ \mathrm{O}(1) & 0,3281(3) & 0,0934(3) & 0,4371(2) & 0,76(4) \\ \mathrm{O}(2) & 0,4453(3) & 0,7097(3) & 0,4674(2) & 0,81(4) \\ \mathrm{O}(3) & 0,1303(3) & 0,9671(3) & 0,3219(2) & 1,01(4) \\ \mathrm{O}(4) & 0,0633(3) & 0,8147(3) & 0,5680(2) & 0,80(4) \\ \mathrm{O}(5) & 0,1650(3) & 0,4064(3) & 0,5469(2) & 1,03(4) \\ \mathrm{O}(6) & 0,3752(4) & 0,3735(3) & 0,7116(2) & 1,22(4) \\ \mathrm{O}(7) & 0,1745(4) & 0,2999(3) & 0,2612(2) & 1,20(4) \\ \mathrm{O}(8) & 0,2869(3) & 0,1025(3) & 0,6722(2) & 0,88(4) \\ \mathrm{O}(9) & 0,1450(4) & 0,8294(3) & 0,8957(2) & 1,61(5) \\ \mathrm{O}(10) & 0,3484(4) & 0,6956(3) & 0,7388(2) & 1,10(4) \\ \mathrm{O}(11) & 0,5334(4) & 0,7192(3) & 0,9003(2) & 1,38(4) \\ \mathrm{O}(12) & 0,2666(4) & 0,4979(3) & 0,9326(2) & 1,65(5) \\ \mathrm{O} w(1) & 0,2888(4) & 0,9523(4) & 0,0960(2) & 1,91(5) \\ \mathrm{O} w(2) & 0,0891(5) & 0,2101(3) & 0,8993(2) & 2,14(5) \\ \mathrm{O} w(3) & 0,1891(4) & 0,6372(3) & 0,2868(2) & 1,44(4) \\ \mathrm{H}^{\prime}(\mathrm{O} w 2) & 0,015(9) & 0,209(7) & 0,951(4) & * \\ \mathrm{H}(\mathrm{O} 6) & 0,441(9) & 0,471(7) & 0,739(4) & * \\ \mathrm{H}^{\prime}(\mathrm{O} w 1) & 0,256(9) & 0,993(7) & 0,020(4) & * \\ \mathrm{H}^{\prime}(\mathrm{O} w 3) & 0,172(9) & 0,521(7) & 0,284(4) & * \\ \mathrm{H}(\mathrm{O} 12) & 0,306(9) & 0,443(7) & 0,989(4) & * \\ \mathrm{H}(\mathrm{O} 7) & 0,285(9) & 0,294(7) & 0,192(4) & * \\ \mathrm{H}^{\prime \prime}(\mathrm{O} w 1) & 0,345(9) & 0,033(7) & 0,097(4) & * \\ \mathrm{H}^{\prime \prime}(\mathrm{O} w 2) & 0,143(9) & 0,309(7) & 0,884(4) & * \\ \mathrm{H}^{\prime \prime}(\mathrm{O} w 3) & 0,293(9) & 0,638(7) & 0,254(4) & *\end{array}$

* Atomes affinés isotropiquement; $B=4,0 \AA^{2}$.

\section{Tableau 2. Distances interatomiques $(\AA)$ et écarts types}

$\begin{array}{llll}\mathrm{Zn}(1)-\mathrm{O}(1) & 2,057(2) & \mathrm{Zn}(2)-\mathrm{O}(2) & 2,080(2) \\ \mathrm{Zn}(1)-\mathrm{O}\left(1^{\mathrm{i}}\right) & 2,123(2) & \mathrm{Zn}(2)-\mathrm{O}(3) & 2,383(2) \\ \mathrm{Zn}(1)-\mathrm{O}(2) & 2,255(2) & \mathrm{Zn}(2)-\mathrm{O}(4) & 2,009(2) \\ \mathrm{Zn}(1)-\mathrm{O}(4) & 2,094(2) & \mathrm{Zn}(2)-\mathrm{O}(5) & 2,133(2) \\ \mathrm{Zn}(1)-\mathrm{O}(8) & 2,138(2) & \mathrm{Zn}(2)-\mathrm{O}\left(5^{\mathrm{i}}\right) & 2,072(2) \\ \mathrm{Zn}(1)-\mathrm{O}(10) & 2,002(2) & \mathrm{Zn}(2)-\mathrm{O} w(3) & 2,067(2) \\ \mathrm{Zn}(3)-\mathrm{O}(3) & 1,981(2) & \mathrm{P}(1)-\mathrm{O}(1) & 1,520(2) \\ \mathrm{Zn}(3)-\mathrm{O}(8) & 2,136(2) & \mathrm{P}(1)-\mathrm{O}(3) & 1,519(2) \\ \mathrm{Zn}(3)-\mathrm{O}(9) & 1,997(2) & \mathrm{P}(1)-\mathrm{O}(4) & 1,528(2) \\ \mathrm{Zn}(3)-\mathrm{O} w(1) & 2,202(2) & \mathrm{P}(1)-\mathrm{O}(7) & 1,571(2) \\ \mathrm{Zn}(3)-\mathrm{O} w(2) & 2,011(2) & & \\ \mathrm{P}(2)-\mathrm{O}(2) & 1,520(2) & \mathrm{P}(3)-\mathrm{O}(9) & 1,514(2) \\ \mathrm{P}(2)-\mathrm{O}(5) & 1,528(2) & \mathrm{P}(3)-\mathrm{O}(10) & 1,527(2) \\ \mathrm{P}(2)-\mathrm{O}(6) & 1,584(2) & \mathrm{P}(3)-\mathrm{O}(11) & 1,531(2) \\ \mathrm{P}(2)-\mathrm{O}(8) & 1,533(2) & \mathrm{P}(3)-\mathrm{O}(12) & 1,581(2)\end{array}$

Code de symétrie: (i) $-x+1,-y,-z+1$; (ii) $-x+1,-y+1,-z+1$. 
Tableau 3. Liaisons par pont-hydrogène

\begin{tabular}{lllccc}
\multicolumn{1}{c}{$\mathrm{D}$ Distances $(\AA)$} & Angle $\left(^{\circ}\right)$ & Distances $(\AA)$ & $\begin{array}{c}\text { Contribution } \\
\text { dans }\end{array}$ \\
$\mathrm{O}-\mathrm{H} \cdots \mathrm{O}$ & $\mathrm{O}-\mathrm{H}$ & $\mathrm{H} \cdots \mathrm{O}$ & $\mathrm{O}-\mathrm{H}-\mathrm{O}$ & $\mathrm{O} \cdots \mathrm{O}$ & l'enchaînement \\
$\mathrm{O} w(2)-\mathrm{H}^{\prime}(\mathrm{O} w 2) \cdots \mathrm{O}(9)$ & $0,74(5)$ & $1,97(5)$ & $170(5)$ & $2,700(3)$ & inter-feuillet \\
$\mathrm{O}(6)-\mathrm{H}(\mathrm{O} 6) \cdots \mathrm{O}(10)$ & $1,07(5)$ & $1,74(4)$ & $131(3)$ & $2,576(2)$ & intra-feuillet \\
$\mathrm{O} w(1)-\mathrm{H}^{\prime}(\mathrm{O} w 1) \cdots \mathrm{O} w(2)$ & $0,95(4)$ & $2,17(4)$ & $144(4)$ & $2,992(3)$ & inter-feuillet \\
$\mathrm{O} w(3)-\mathrm{H}^{\prime}(\mathrm{O} w 3) \cdots \mathrm{O}(7)$ & $0,93(5)$ & $1,81(5)$ & $171(4)$ & $2,726(3)$ & intra-feuillet \\
$\mathrm{O}(12)-\mathrm{H}^{\prime}(\mathrm{O} 12) \cdots \mathrm{O}(11)$ & $0,76(4)$ & $1,89(4)$ & $163(5)$ & $2,630(3)$ & inter-feuillet \\
$\mathrm{O}(7)-\mathrm{H}(\mathrm{O} 7) \cdots \mathrm{O}(11)$ & $1,03(4)$ & $1,51(4)$ & $173(4)$ & $2,538(2)$ & intra-feuillet \\
$\mathrm{O} w(1)-\mathrm{H}^{\prime \prime}(\mathrm{O} w 1) \cdots \mathrm{O}(11)$ & $0,78(5)$ & $2,18(5)$ & $173(5)$ & $2,956(3)$ & intra-feuillet \\
$\mathrm{O} w(2)-\mathrm{H}^{\prime \prime}(\mathrm{O} w 2) \cdots \mathrm{O}(12)$ & $0,85(5)$ & $2,03(5)$ & $151(4)$ & $2,801(3)$ & intra-feuillet \\
$\mathrm{O} w(3)-\mathrm{H}^{\prime \prime}(\mathrm{O} w 3) \cdots \mathrm{O}(6)$ & $0,73(5)$ & $2,22(5)$ & $137(4)$ & $2,793(3)$ & intra-feuillet
\end{tabular}

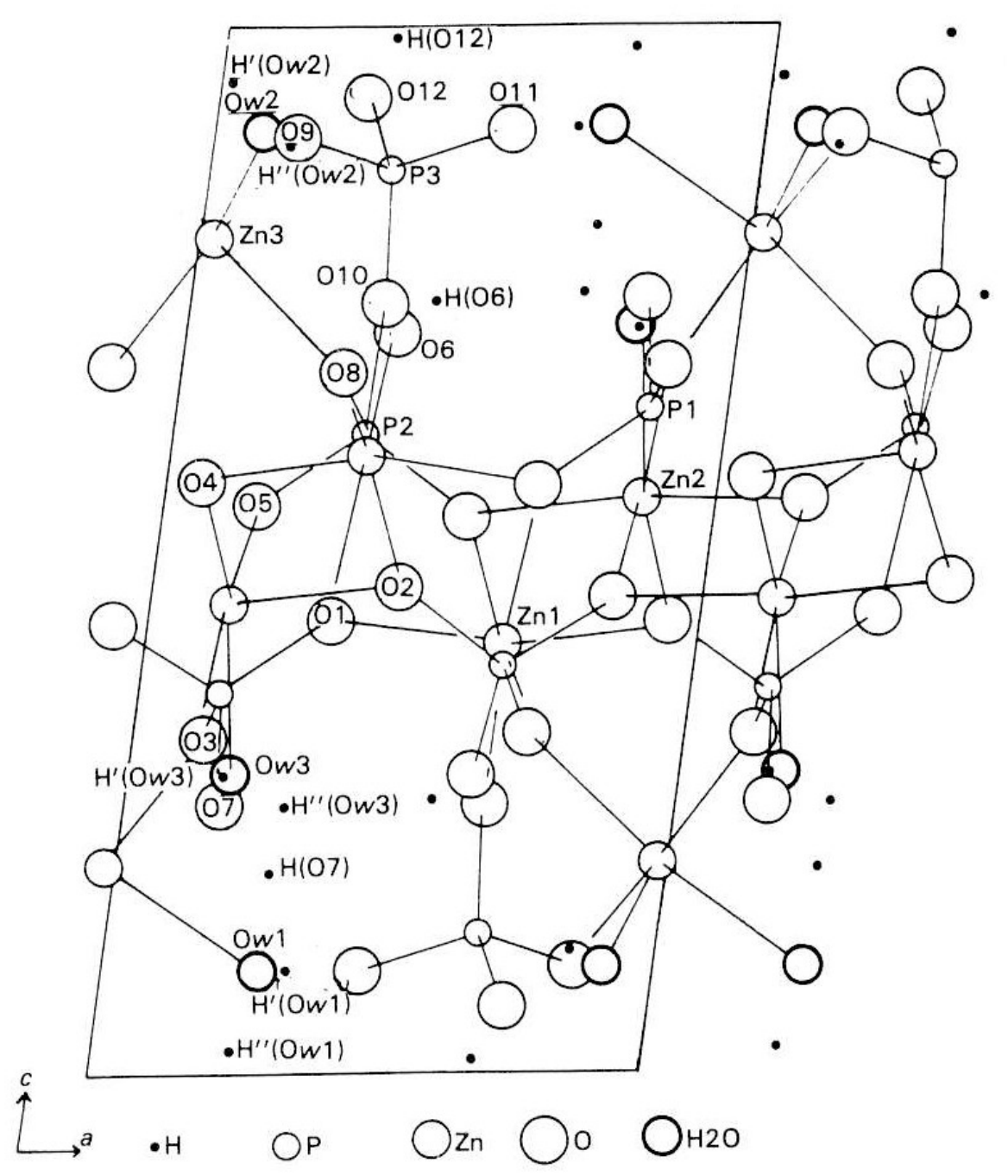

Fig. 1. Projection de la structure suivant $\mathbf{b}$. 


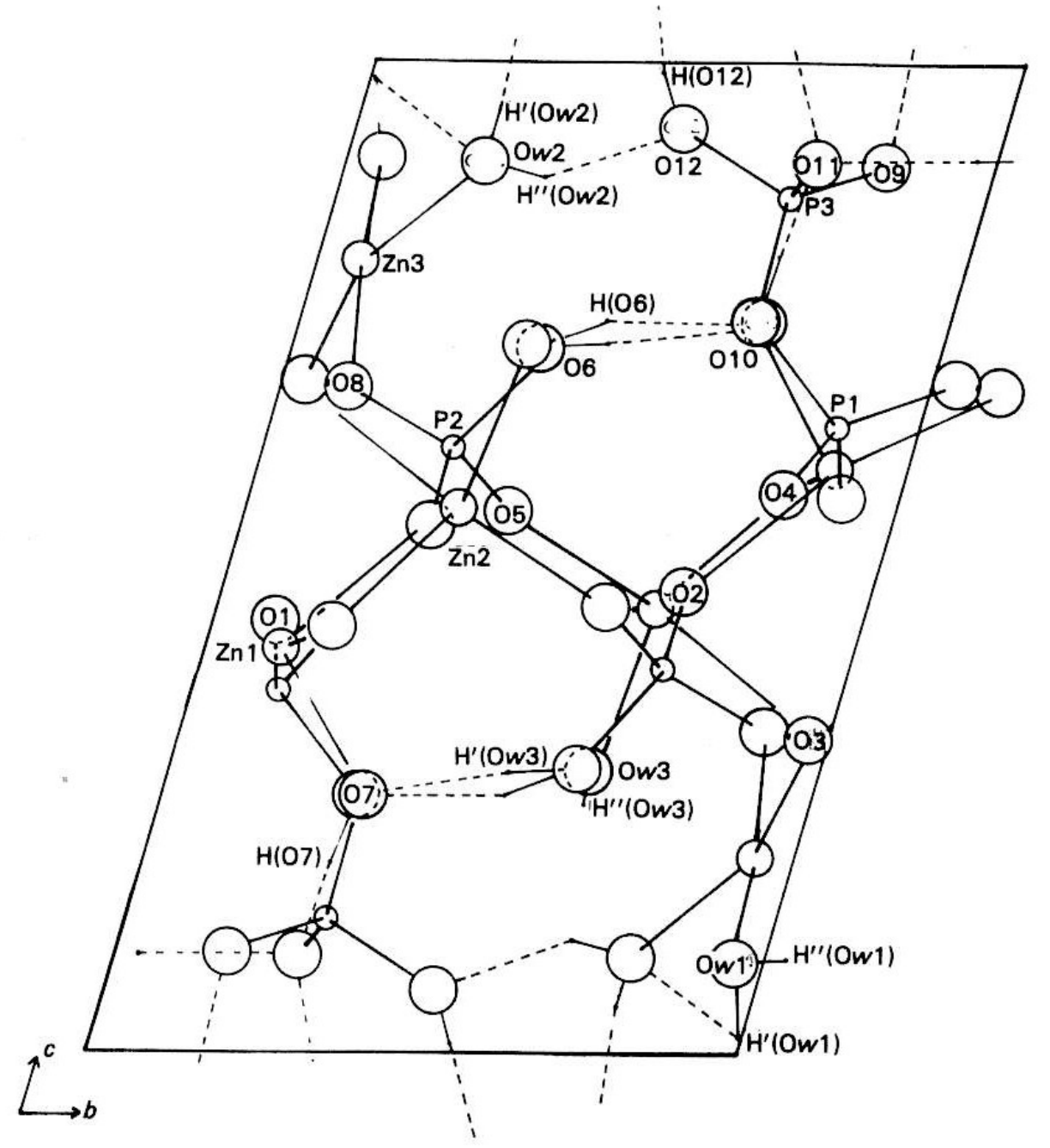

Fig. 2. Projection de la structure suivant a. 